\title{
Effect of Ethyl Pyruvate on Paclitaxel-Induced Neuropathic Pain in Rats
}

\author{
Department of Anesthesiology and Pain Medicine, Asan Medical Center, \\ University of Ulsan College of Medicine, Seoul, Korea \\ Seong Soo Choi, MD, Won Uk Koh, MD, Jae Sik Nam, MD, \\ Jin Woo Shin, MD, Jeong Gill Leem, MD, and Jeong Hun Suh, MD
}

\section{Background:}

Although paclitaxel is a widely used chemotherapeutic agent for the treatment of solid cancers, side effects such as neuropathic pain lead to poor compliance and discontinuation of the therapy. Ethyl pyruvate (EP) is known to have analgesic effects in several pain models and may inhibit apoptosis. The present study was designed to investigate the analgesic effects of $\mathrm{EP}$ on mechanical allodynia and apoptosis in dorsal root ganglion (DRG) cells after paclitaxel administration.

\section{Methods:}

Rats were randomly divided into 3 groups: 1) a control group, which received only vehicle; 2) a paclitaxel group, which received paclitaxel; and 3) an EP group, which received EP after paclitaxel administration. Mechanical allodynia was tested before and at 7 and 14 days after final paclitaxel administration. Fourteen days after paclitaxel treatment, DRG apoptosis was determined by activated caspase-3 immunoreactivity (IR).

\section{Results:}

Post-treatment with EP did not significantly affect paclitaxel-induced allodynia, although it tended to slightly reduce sensitivities to mechanical stimuli after paclitaxel administration. After paclitaxel administration, an increase in caspase-3 IR in DRG cells was observed, which was co-localized with NF200-positive myelinated neurons. Post-treatment with EP decreased the paclitaxel-induced caspase-3 IR. Paclitaxel administration or post-treatment with EP did not alter the glial fibrillary acidic protein IRs in DRG cells.

\section{Conclusions:}

Inhibition of apoptosis in DRG neurons by EP may not be critical in paclitaxel-induced mechanical allodynia. (Korean J Pain 2013; 26: 135-141)

\section{Key Words:}

allodynia, apoptosis, dorsal root ganglion, ethyl pyruvate, paclitaxel.

Received November 22, 2012. Revised December 14, 2012. Accepted December 17, 2012.

Correspondence to: Jeong Hun Suh, MD

Department of Anesthesiology and Pain Medicine, Asan Medical Center, University of Ulsan College of Medicine, 388-1 Pungnap 2-dong, Songpa-gu, Seoul 138-736, Korea

Tel: +82-2-3010-3868; Fax: +82-2-3010-6790, E-mail: paindrsuh@gmail.com

(a) This is an open-access article distributed under the terms of the Creative Commons Attribution Non-Commercial License (http:// creativecommons.org/licenses/by-nc/3.0/), which permits unrestricted non-commercial use, distribution, and reproduction in any medium, provided the original work is properly cited.

Copyright (c) The Korean Pain Society, 2013 


\section{INTRODUCTION}

Paclitaxel (Taxol) is a widely used chemotherapeutic agent for the treatment of solid cancers. Its anti-neoplastic activity is known to increase microtubule stabilization [1]. However, it has several side effects, including peripheral neuropathy and allodynia, which lead to poor compliance and discontinuation of the therapy $[2,3]$. The induction of peripheral neuropathy is the main dose-limiting side effect of paclitaxel treatment [4]. Although the exact mechanism of paclitaxel-induced neuropathic pain is not fully understood, several reports have shown that the derangement of nerve fiber endings may play a role in the development of this pain $[5,6]$. Sensory neurons and satellite cells located at the dorsal root ganglion (DRG) are altered by paclitaxel treatment [7-9]. In addition, it is well known that apoptosis is evident in DRG neurons following nerve injury, which may induce allodynia [10-12]. Neurotoxic effects in DRG cells after paclitaxel treatment have also been reported $[7,8]$.

Ethyl pyruvate (EP) is a stable, lipophilic derivative of pyruvic acid with known anti-inflammatory and antioxidant properties. It has been reported that administration of EP may reduce brain and spinal cord injuries in cerebral and spinal cord ischemic/reperfusion-induced injury models and spinal cord compression injury models [13-15]. EP is also known to inhibit apoptosis in various models of organ injury [15-17] and have analgesic effects on spinal nerve ligation- or inflammation-induced painful behaviors [18-20]. For example, we recently reported that EP has a strong anti-allodynic effect that acts through the inhibition of tumor necrosis factor (TNF) $-\alpha$ expression and apoptosis in the DRG after spinal nerve ligation injury [18]. However, the analgesic effect of EP has not been evaluated carefully in chemoneuropathic pain, such as paclitaxel-induced allodynia.

Therefore, the specific aim of the present study was to evaluate the analgesic effect of EP on mechanical allodynia and apoptosis in DRG cells after paclitaxel administration.

\section{MATERIALS AND METHODS}

This study was reviewed and approved by the Institutional Animal Care and Use Committee (IACUC) of the Asan Institute for Life Sciences, Asan Medical Center. The committee follows the Institute of Laboratory Animal
Resources (ILAR) guide.

\section{Experimental animals}

Male Sprague-Dawley rats (160-180 g) were housed 3 per cage in a room maintained at $21 \pm 1.0^{\circ} \mathrm{C}$ with an alternating $12 \mathrm{~h}$ light-dark cycle. Rats were provided with water and food ad libitum and permitted to acclimate for at least 3 days prior to their use in experiments. The animals were allowed to adapt to the laboratory for at least $2 \mathrm{~h}$ before testing, and they were used only once. To reduce variation, all experiments were performed during the light phase of the cycle (10:00-17:00).

\section{Experimental protocol and drug administration}

The rats were randomly divided into 3 groups (21 rats per group): 1) the control group, which received only vehicle; 2) the paclitaxel group, which received paclitaxel; and 3) the EP group, which received EP after paclitaxel administration. Paclitaxel (Taxol ${ }^{\circledR}$; Bristol-Myers-Squibb, Montreal, Canada; $6 \mathrm{mg} / \mathrm{ml}$ ) was diluted with saline to a concentration of $2 \mathrm{mg} / \mathrm{ml}$ and injected intraperitoneally at a dose of 2 $\mathrm{mg} / \mathrm{kg}$ on 4 alternate days (days 0,2 , 4, and 6), as previously described [5,6,21,22]. EP (98\% pure; Sigma-Aldrich Co., St. Louis, MO, USA) was dissolved in normal saline to a concentration of $10 \mathrm{mg} / \mathrm{ml}$ and injected intraperitoneally at a dose of $50 \mathrm{mg} / \mathrm{kg}$ on 6 consecutive days after final paclitaxel administration. The dose of EP was chosen based on preliminary studies and previous publications [18]. In addition, previous studies have evaluated the therapeutic effect of EP administered repeatedly in several animal disease models [23,24]. Thus, following previous reports, we repeatedly administered EP on 6 consecutive days but with a modification. During EP administration, the control and paclitaxel groups were treated with the vehicle for EP (normal saline) on the same 6 consecutive days.

Behavioral tests were performed before and at 7 and 14 days after final administration of paclitaxel in order to measure behavioral responses to mechanical stimuli. DRG apoptosis was evaluated by immunohistochemical detection of activated caspase-3 14 days after final paclitaxel administration. All testing was performed by 1 examiner who was blind to the study groups.

\section{Behavioral tests}

Behavioral tests were performed during the same time period on each testing day (10:00 AM to 4:00 PM). Rats 
were positioned in individual plastic cages with wire-mesh bottoms and permitted to acclimate for 20 minutes. Baseline testing was performed before drug administration to verify that the animal responses were normal. To test for mechanical allodynia, a von Frey filament (Stoelting, Wood Dale, IL, USA) was used to stimulate the plantar surface of the left hind foot. Eight calibrated von Frey filaments (0.41, 0.70, $1.20,2.00,3.63,5.50,8.50$, and $15.10 \mathrm{~g}$ ) were sequentially applied to the plantar surface of the foot (in ascending or descending order) and gently pressed against the foot. Rapid withdrawal or flinching was interpreted as a positive response, in which case the next-lightest filament was tested; a negative response led to the application of the nextheaviest filament. Determination of the $50 \%$ withdrawal threshold was made using the up-down method, as previously described [25-27].

\section{Immunohistochemistry}

The histological findings in the 21 rats used in the behavioral tests were also analyzed ( $n=7$ rats per group), as previously described $[12,18]$. Briefly, under anesthesia, the rats were transcardially perfused with $4 \%$ buffered paraformaldehyde 14 days after final paclitaxel administration. The L5 DRG was collected and subsequently fixed in $4 \%$ paraformaldehyde, embedded in paraffin, and sectioned to a thickness of $4 \mu \mathrm{m}$. After blocking with $5 \%$ goat serum, $0.3 \%$ Triton $X-100$, and $1 \%$ bovine serum albumin in phosphate-buffered saline (PBS-T), double-label immunofluorescence was performed by incubating the paraffin sections overnight at $4^{\circ} \mathrm{C}$ with antibodies against cleaved caspase-3 (Asp 175) (1:200; Cell Signaling Technology Inc., Danvers, MA, USA) and glial fibrillary acidic protein (GFAP) (1 :200; Cell Signaling) or the N52 clone of neurofilament 200 (NF200) (1 :200; SigmaAldrich), followed by incubation with Alexa Fluor 555 goat anti-rabbit IgG (red; 1 : 500; Invitrogen, Carlsbad, CA, USA) and Alexa Fluor 488 goat anti-mouse IgG (green; 1 : 500; Invitrogen) for 2 hours at $20^{\circ} \mathrm{C}$. The immunostained DRG sections were observed using a confocal microscope. Two sections were used in each evaluation to count the number of NF200-immunoreactive (IR), GFAP-IR, and double-labeled cells at $400 \times$ magnification. The percentage of double-labeled cells in each DRG was calculated by dividing the number of double-labeled cells by the total number of NF200-IR or GFAP-IR cells within the field and multiplying the result by 100 .

\section{Statistical analysis}

The data are presented as the means \pm standard error. Behavioral data (withdrawal threshold to mechanical stimuli) were evaluated by 2-way repeated measures analysis of variance. The percentage of double-labeled cells among the DRG cells was analyzed using the KruskalWallis test followed by the Mann-Whitney U-test to evaluate the between-group differences. $P$ values less than 0.05 were considered significant.

\section{RESULTS}

In the present study, normal weight gain was observed in all groups of animals during the testing periods (data not shown), and no animals died during paclitaxel therapy, similar to previous reports [22,28,29].

\section{Effect of EP on paclitaxel-induced mechanical allodynia}

The baseline threshold to evoke a withdrawal response to mechanical stimuli in all rats before drug administration was $15.10 \mathrm{~g}$ (maximal mechanical stimulus). However, as

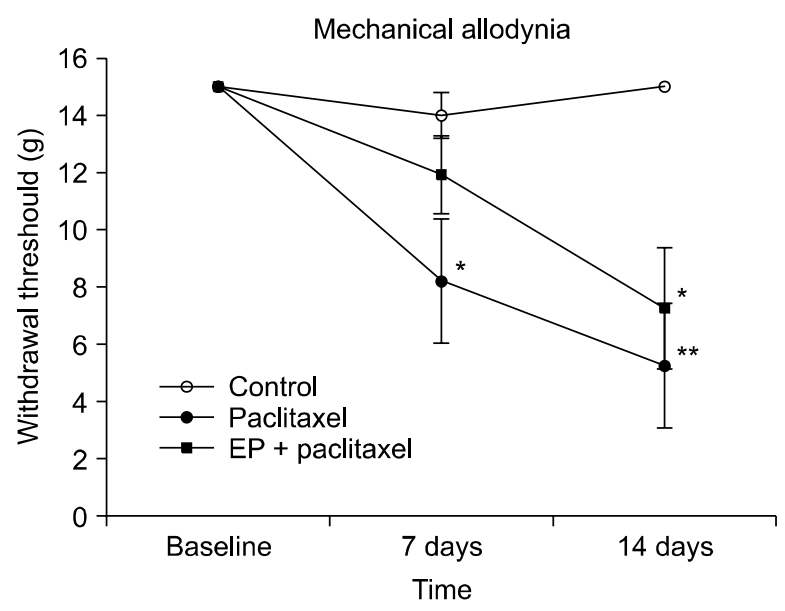

Fig. 1. The effect of ethyl pyruvate (EP) on paclitaxelinduced mechanical allodynia in rats. Groups of rats were injected i.p. with either vehicle (control) or paclitaxel (2 $\mathrm{mg} / \mathrm{kg}$ on 4 alternate days). Another group of rats was treated i.p. with EP (50 mg/ $\mathrm{kg}$ on 6 consecutive days) after final paclitaxel administration. Mechanical allodynia was tested before and at 7 and 14 days after final paclitaxel administration by application of von Frey filaments to the surface of the hind paw. The vertical bars indicate the standard error of the mean. The number of animals used for each group was $7{ }^{*} P<0.05$ and ${ }^{*} P P<0.01$ compared with the control group). 
shown in Fig. 1, the withdrawal threshold to mechanical stimuli was reduced significantly at 7 and 14 days after paclitaxel administration $(P=0.023$ for 7 days, $P=0.004$ for 14 days). Post-treatment with EP did not significantly

\section{A}

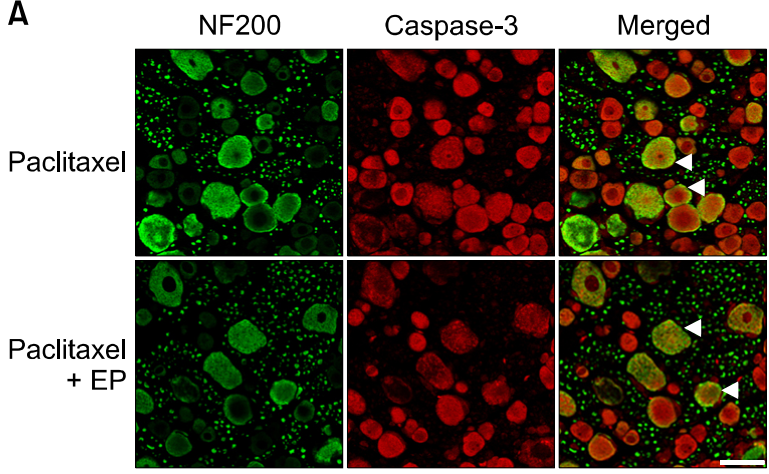

\section{B}

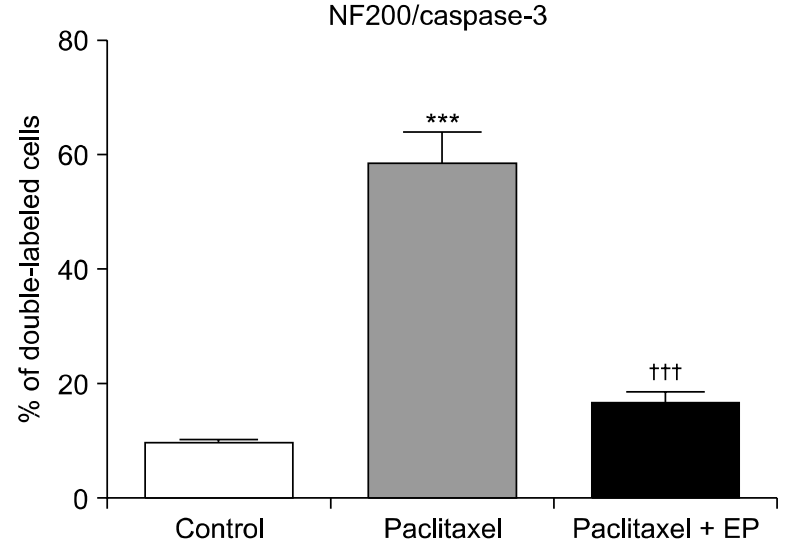

Fig. 2. The representative double immunofluorescence staining and quantitative analysis of activated caspase-3 and neurofilament 200 (NF200) in the rat dorsal root ganglion (DRG). Groups of rats were injected i.p. with either vehicle (control) or paclitaxel $(2 \mathrm{mg} / \mathrm{kg}$ on 4 alternate days). Another group of rats was treated i.p. with either vehicle or ethyl pyruvate (EP, $50 \mathrm{mg} / \mathrm{kg}$ on 6 consecutive days) after final paclitaxel administration. Fourteen days after paclitaxel treatment, L5 DRG samples were immunostained with caspase-3, an indicator of apoptosis, and NF200, a marker of myelinated neurons. The co-localization of caspase- 3 was visualized in a merged image. The arrow heads indicate where activated caspase- 3 is co-localized with NF200. The scale bar represents $50 \mu \mathrm{m}$ (A). The NF200 positive neurons that co-localized with caspase-3 were counted and are expressed as a percentage of the total neurons per section ${ }^{\star * \star} P<0.001$ compared with the control group, and ${ }^{\dagger \dagger} P<0.001$ compared with paclitaxel group). Error bars represent SEM (B). affect paclitaxel-induced allodynia $(P=0.071$ for 7 days, $P=0.088$ for 14 days), although it tended to slightly reduce sensitivities to mechanical stimuli after paclitaxel administration (Fig. 1).

2. Effect of EP on NF200 and caspase-3 IRs in paclitaxel-induced neuropathy

The immunohistochemical analysis at 14 days after $\mathrm{pa}^{-}$ clitaxel administration showed an increase in caspase-3 IR in the DRG cells, which was co-localized with NF200- positive neurons (Fig. 2A). Moreover, post-treatment with EP decreased the paclitaxel-induced caspase-3 IR, shown Fig. 2A. The percentage of NF200-positive DRG neurons co-localized with caspase-3 at 14 days after paclitaxel administration was significantly increased compared with the vehicle-treated control group $(P<0.001$, Fig. $2 \mathrm{~B})$. The paclitaxel-induced caspase-3 IR in the DRG neurons was inhibited by post-treatment with EP ( $P<0.001$, Fig. 2B).

3. Effect of EP on GFAP and caspase-3 IRs in paclitaxelinduced neuropathy

An immunohistochemical analysis showed that paclitaxel administration did not alter the GFAP IRs in the DRG com-



Fig. 3. The representative double immunofluorescence staining of activated caspase- 3 and glial fibrillary acidic protein (GFAP) in the rat dorsal root ganglion (DRG). Groups of rats were injected i.p. with either vehicle (control) or paclitaxel ( $2 \mathrm{mg} / \mathrm{kg}$ on 4 alternate days). Another group of rats was treated i.p. with either vehicle or ethyl pyruvate (EP, $50 \mathrm{mg} / \mathrm{kg}$ on 6 consecutive days) after final paclitaxel administration. Fourteen days after paclitaxel treatment, L5 DRG samples were immunostained with caspase-3, an indicator of apoptosis, and GFAP, a marker of activated astrocytes. Neither paclitaxel nor post-treatment with EP affected GFAP- or caspase-3 IRs in DRG. The scale bar represents $50 \mu \mathrm{m}$. 
pared with the vehicle-treated control group (data not shown). Additionally, post-treatment with EP had no effect on the GFAP and caspase-3 IRs located in the DRG (Fig. 3).

\section{DISCUSSION}

Systemic administration of paclitaxel can induce neuropathy, mechanical allodynia, or thermal hyperalgesia $[2,3]$. Intraperitoneal injection of paclitaxel at a dose of 2 $\mathrm{mg} / \mathrm{kg}$ on 4 alternate days is believed to be a reliable model of peripheral neuropathy $[22,29]$. The pathophysiology of paclitaxel-induced neuropathic pain appears to involve changes in the primary sensory nerve, DRG, and spinal cord levels [8,9,21,28,30,31]. For example, in a previous study, myelinated A-fiber hypersensitization was observed at 1 and 2 weeks after paclitaxel treatment [21]. Another study found significant increases of atypical (swollen and vacuolated) mitochondria in both $\mathrm{C}$-fibers and myelinated axons of paclitaxel-treated nerves [28]. Paclitaxel may also induce apoptosis in DRG cells and cortical neurons [7,8,32,33]. Melli et al. [7] found that paclitaxel caused early mitochondrial impairment, with loss of membrane potential and induction of autophagic vacuoles, in the primary cultures of DRG sensory neurons [7]. Another in vitro studies have shown that the neurotoxic and apoptotic effects of paclitaxel were dose- and time-dependent in DRG explants and cultured neuronal cells $[8,32,33]$.

On the other hand, it is relatively well known that apoptosis may be evident in DRG neurons following nerve injury, which may induce allodynia [10-12]. Caspase-3 can induce the proteolytic cleavage of many key proteins, and its expression is regarded as a distinct indicator of apoptosis [34,35]. In the present study, we found increased caspase-3 IR in DRG cells, which was co-localized with NF200-positive myelinated neurons 14 days after paclitaxel administration. Moreover, post-treatment with EP decreased the paclitaxel-induced caspase-3 IR. However, EP did not affect the paclitaxel-induced mechanical allodynia significantly, although post-treatment with EP tended to slightly reduce the withdrawal threshold to mechanical stimuli after paclitaxel administration. Although we did not investigate the dose-dependent effects of EP in the present study, the possibility of an anti-allodynic effect from EP administered at doses higher than $50 \mathrm{mg} / \mathrm{kg}$ cannot be ruled out. Recently, we reported that EP has a strong anti-allodynic effect that acts through the inhibition of TNF- $\alpha$ expression and apoptosis in the DRG after spinal nerve ligation injury [18]. Further studies to determine the exact time and dose of EP administration are needed.

Interestingly, the present study showed that paclitaxel administration (2 $\mathrm{mg} / \mathrm{kg}$ on 4 alternate days) did not alter GFAP IRs in the DRG. Contrary to our present findings, a previous study on cellular pathology in DRG after paclitaxel treatment reported that the GFAP IR levels in the DRG were increased in paclitaxel-treated rats compared with vehicle-treated rats from days 6 through 10 after final administration of paclitaxel [9]. Because these researchers injected paclitaxel intravenously into the tail vein of rats at a dose of $18 \mathrm{mg} / \mathrm{kg}$ twice (on days 0 and 3), this discrepancy may be explained in part by the differences in duration and dose of paclitaxel administration. Therefore, these findings suggest that astrocytes located in the DRG may have a time-specific or dose-specific involvement in paclitaxel-induced neuropathic pain. In addition, paclitaxel evokes the release of pro-inflammatory cytokines, including TNF- $\alpha[36,37]$. Pro-inflammatory cytokines have an important role in the pathogenesis of painful peripheral neuropathies and neuropathic pain (for a review, see $[38,39])$. Several studies have reported the involvement of spinal glial cells and cytokines in the pathogenesis of paclitaxel-induced neuropathic pain $[40,41]$. Burgos and colleagues observed a strong microglial and astroglial activation and induction of pro-inflammatory cytokines in the lumbar spinal cord after paclitaxel administration [41]. In the present study, apoptosis of DRG neurons, but not mechanical allodynia, was inhibited by post-treatment with EP. Therefore, this result cannot exclude the possibility that spinal glial cells have a more important role in the development of paclitaxel-induced neuropathic pain than DRG astrocytes, although we did not examine the involvement of glial cells located in the spinal cord and the pro-inflammatory cytokine levels.

In conclusion, inhibition of apoptosis of DRG neurons by EP may not be critical in paclitaxel-induced mechanical allodynia. Further studies are needed to elucidate the exact time and dose of EP administration in the paclitaxel-induced neuropathic pain model.

\section{REFERENCES}

1. Jordan MA, Wilson L. Microtubules as a target for anticancer drugs. Nat Rev Cancer 2004; 4: 253-65. 
2. Forsyth PA, Balmaceda C, Peterson K, Seidman AD, Brasher $P$, DeAngelis $L M$. Prospective study of paclitaxel-induced peripheral neuropathy with quantitative sensory testing. J Neurooncol 1997; 35: 47-53.

3. Postma TJ, Vermorken JB, Liefting AJ, Pinedo HM, Heimans JJ. Paclitaxel-induced neuropathy. Ann Oncol 1995; 6: 489-94.

4. Cavaletti G, Bogliun G, Marzorati L, Zincone A, Marzola M, Colombo N, et al. Peripheral neurotoxicity of taxol in patients previously treated with cisplatin. Cancer 1995; 75: $1141-50$.

5. Boyette-Davis J, Xin W, Zhang H, Dougherty PM. Intraepidermal nerve fiber loss corresponds to the development of taxol-induced hyperalgesia and can be prevented by treatment with minocycline. Pain 2011; 152: 308-13.

6. Siau C, Xiao W, Bennett GJ. Paclitaxel- and vincristineevoked painful peripheral neuropathies: loss of epidermal innervation and activation of Langerhans cells. Exp Neurol 2006; 201: 507-14.

7. Melli G, Taiana M, Camozzi F, Triolo D, Podini P, Quattrini A, et al. Alpha-lipoic acid prevents mitochondrial damage and neurotoxicity in experimental chemotherapy neuropathy. Exp Neurol 2008; 214: 276-84.

8. Scuteri A, Nicolini G, Miloso M, Bossi M, Cavaletti G, Windebank AJ, et al. Paclitaxel toxicity in post-mitotic dorsal root ganglion (DRG) cells. Anticancer Res 2006; 26: 1065-70.

9. Peters CM, Jimenez-Andrade JM, Kuskowski MA, Ghilardi JR, Mantyh PW. An evolving cellular pathology occurs in dorsal root ganglia, peripheral nerve and spinal cord following intravenous administration of paclitaxel in the rat. Brain Res 2007; 1168: 46-59.

10. Zimmermann M. Pathobiology of neuropathic pain. Eur J Pharmacol 2001; 429: 23-37.

11. Sekiguchi M, Sekiguchi Y, Konno S, Kobayashi H, Homma $Y$, Kikuchi S. Comparison of neuropathic pain and neuronal apoptosis following nerve root or spinal nerve compression. Eur Spine J 2009; 18: 1978-85.

12. Kim SH, Nam JS, Choi DK, Koh WW, Suh JH, Song JG, et al. Tumor necrosis factor-alpha and apoptosis following spinal nerve ligation injury in rats. Korean J Pain 2011; 24: 185-90.

13. Yu YM, Kim JB, Lee KW, Kim SY, Han PL, Lee JK. Inhibition of the cerebral ischemic injury by ethyl pyruvate with a wide therapeutic window. Stroke 2005; 36: 2238-43.

14. Wang Q, Ding Q, Zhou Y, Gou X, Hou L, Chen S, et al. Ethyl pyruvate attenuates spinal cord ischemic injury with a wide therapeutic window through inhibiting high-mobility group box 1 release in rabbits. Anesthesiology 2009; 110: 127986.

15. Genovese T, Esposito E, Mazzon E, Di Paola R, Meli R, Caminiti $R$, et al. Beneficial effects of ethyl pyruvate in a mouse model of spinal cord injury. Shock 2009; 32: 217-27.

16. Guo J, Zhang K, Ji Y, Jiang X, Zuo S. Effects of ethyl pyruvate on myocardial apoptosis and expression of $\mathrm{Bcl}-2$ and $\mathrm{Bax}$ proteins after ischemia-reperfusion in rats. J Huazhong Univ Sci Technolog Med Sci 2008; 28: 281-3.

17. Tsung A, Kaizu T, Nakao A, Shao L, Bucher B, Fink MP, et al. Ethyl pyruvate ameliorates liver ischemia-reperfusion injury by decreasing hepatic necrosis and apoptosis. Transplantation 2005; 79: 196-204.

18. Choi DK, Leem JG, Shin JW, Suh JH. Effects of ethyl pyruvate on allodynia, TNF- $\alpha$ expression, and apoptosis in the dorsal root ganglion after spinal nerve ligation injury. Korean J Pain 2012; 25: 213-20.

19. Lee MJ, Jang M, Jung HS, Kim SH, Cho $H$. Ethyl pyruvate attenuates formalin-induced inflammatory nociception by inhibiting neuronal ERK phosphorylation. Mol Pain 2012; 8: 40.

20. Jacobs CC, Holcombe SJ, Cook VL, Gandy JC, Hauptman $J G$, Sordillo LM. Ethyl pyruvate diminishes the inflammatory response to lipopolysaccharide infusion in horses. Equine Vet J 2012 [in press].

21. Matsumoto M, Inoue M, Hald A, Xie W, Ueda H. Inhibition of paclitaxel-induced A-fiber hypersensitization by gabapentin. J Pharmacol Exp Ther 2006; 318: 735-40.

22. Polomano RC, Mannes AJ, Clark US, Bennett GJ. A painful peripheral neuropathy in the rat produced by the chemotherapeutic drug, paclitaxel. Pain 2001; 94: 293-304.

23. Choi JS, Lee MS, Jeong JW. Ethyl pyruvate has a neuroprotective effect through activation of extracellular signal-regulated kinase in Parkinson's disease model. Biochem Biophys Res Commun 2010; 394: 854-8.

24. Yang R, Shaufl AL, Killeen ME, Fink MP. Ethyl pyruvate ameliorates liver injury secondary to severe acute pancreatitis. J Surg Res 2009; 153: 302-9.

25. Li Y, Dorsi MJ, Meyer RA, Belzberg AJ. Mechanical hyperalgesia after an L5 spinal nerve lesion in the rat is not dependent on input from injured nerve fibers. Pain 2000; 85: 493-502.

26. Choi Jl, Kim WM, Yoon MH, Lee HG. Antiallodynic effect of thalidomide and morphine on rat spinal nerve ligation-induced neuropathic pain. Korean J Pain 2010; 23: 172-8.

27. Chaplan SR, Bach FW, Pogrel JW, Chung JM, Yaksh TL. Quantitative assessment of tactile allodynia in the rat paw. J Neurosci Methods 1994; 53: 55-63.

28. Flatters SJ, Bennett GJ. Studies of peripheral sensory nerves in paclitaxel-induced painful peripheral neuropathy: evidence for mitochondrial dysfunction. Pain 2006; 122: 245-57.

29. Pascual D, Goicoechea C, Suardíaz M, Martín Ml. A cannabinoid agonist, WIN 55,212-2, reduces neuropathic nociception induced by paclitaxel in rats. Pain 2005; 118 : 23-34. 
30. Xiao WH, Bennett GJ. Chemotherapy-evoked neuropathic pain: abnormal spontaneous discharge in A-fiber and C-fiber primary afferent neurons and its suppression by acetyl-L-carnitine. Pain 2008; 135: 262-70.

31. Zhang J, Tuckett RP. Comparison of paclitaxel and cisplatin effects on the slowly adapting type I mechanoreceptor. Brain Res 2008; 1214: 50-7.

32. Figueroa-Masot XA, Hetman M, Higgins MJ, Kokot N, Xia Z. Taxol induces apoptosis in cortical neurons by a mechanism independent of Bcl-2 phosphorylation. J Neurosci 2001; 21: 4657-67.

33. Jang HJ, Hwang S, Cho KY, Kim do K, Chay KO, Kim JK. Taxol induces oxidative neuronal cell death by enhancing the activity of NADPH oxidase in mouse cortical cultures. Neurosci Lett 2008; 443: 17-22.

34. Chen M, Wang J. Initiator caspases in apoptosis signaling pathways. Apoptosis 2002; 7: 313-9.

35. Joseph EK, Levine JD. Caspase signalling in neuropathic and inflammatory pain in the rat. Eur J Neurosci 2004; 20: 2896-902.

36. Burkhart CA, Berman JW, Swindell CS, Horwitz SB. Relationship between the structure of taxol and other taxanes on induction of tumor necrosis factor-alpha gene expression and cytotoxicity. Cancer Res 1994; 54: 5779-82.

37. Manthey CL, Brandes ME, Perera PY, Vogel SN. Taxol increases steady-state levels of lipopolysaccharide-inducible genes and protein-tyrosine phosphorylation in murine macrophages. J Immunol 1992; 149: 2459-65.

38. Kiguchi N, Kobayashi $Y$, Kishioka S. Chemokines and cytokines in neuroinflammation leading to neuropathic pain. Curr Opin Pharmacol 2012; 12: 55-61.

39. Wang XM, Lehky TJ, Brell JM, Dorsey SG. Discovering cytokines as targets for chemotherapy-induced painful peripheral neuropathy. Cytokine 2012; 59: 3-9.

40. Zhang H, Yoon SY, Zhang H, Dougherty PM. Evidence that spinal astrocytes but not microglia contribute to the pathogenesis of Paclitaxel-induced painful neuropathy. J Pain 2012; 13: 293-303.

41. Burgos E, Gómez-Nicola D, Pascual D, Martín MI, NietoSampedro M, Goicoechea C. Cannabinoid agonist WIN 55,212-2 prevents the development of paclitaxel-induced peripheral neuropathy in rats. Possible involvement of spinal glial cells. Eur J Pharmacol 2012; 682: 62-72. 